\title{
Los conceptos foucaultianos de discurso y la producción académica de la educación física brasileña: diálogos, problematizaciones y devenires \\ The Foucaultian concepts of discourse and the academic production of Brazilian physical education: dialogues, problematizations and evolutions \\ *Santiago Pich, **Miguel Vicente Pedraz \\ *Universidad Federal de Santa Catarina (Brasil), **Universidad de León
}

Resumen. El artículo propone una discusión del concepto de discurso en el pensamiento de Michel Foucault en sus tres dominios (sersaber, ser-poder y ser-consigo), entendiendo tal categoría como una de las más importantes de su «caja de herramientas». A partir de esta discusión se dialoga con la producción en el campo de la educación física brasileña; el propósito es comprender los modos de recepción de la noción foucaultiana de discurso en sus diferentes dominios y proponer nuevos problemas posibles para el campo de la educación física. Se constata que, aunque la presencia del pensamiento de Foucault y de su concepto de discurso en el campo académico de la educación física brasileña es intensa y fecunda, el tercer dominio, el ser-consigo, permanece casi inexplorado. Asimismo, se pone de relieve el gran potencial de esta categoría para el desarrollo de nuevas problematizaciones en el campo de la educación física.

Palabras clave: Michel Foucault, Discurso, Educación Física brasileña.

\begin{abstract}
The article proposes a discussion on the concept of discourse in its three domains (being-knowing, being-being and being-with oneself) based on Michel Foucault's thought, understanding this category as one of the most important of his «toolbox». Starting from this discussion, a dialogue develops with the production in the field of Brazilian physical education; the purpose is to understand the ways Foucault's notion of discourse in its different domains is received, and to propose new possible problems for the field of physical education. Although the presence of Foucault's thought and his concept of discourse in the academic field of Brazilian physical education is intense and fruitful, the third domain, being-with-oneself, remains almost unexplored. Likewise, the great potential of this category for the development of new problematizations in the field of physical education is highlighted.
\end{abstract}

Keywords: Michel Foucault, Discourse, Brazilian Physical Education.

\section{Consideraciones iniciales}

La abundante y variada producción teórica de Michel Foucault ha sido un campo fértil de problematizaciones en muy diversos dominios del saber. Ya sea como «foucaultistas» o como «foucaultianos», según la categorización de Vázquez (2010, pp.8-9), es decir, como descifradores de su obra o como investigadores que husmean en la caja de herramientas en busca de conceptos que aplicar a sus particulares intereses, Foucault sigue inspirando a muchos en la forma de analizar la realidad y tal vez en la conformación de sus pesquisas.

En el dominio epistemológico de la Educación Física ha sido invocado de forma recurrente. Particularmente, los adeptos de las corrientes pedagógicas más críticas llevan tiempo aplicando algunas de las más genuinas categorías genealógicas de Foucault como disciplina, sujeto, poder, saber, dispositivo, anatomía política, biopolítica etc., toda vez que el trabajo con o sobre el cuerpo en el que consiste la acción físico-educativa dentro de la institución escolar se ofrece literal y metafóricamente expedito para ser comprendido (o explicado) a partir de dichas categorías; al menos para ser problematizado allí donde las justificaciones del discurso tecnoburocrático de la materia, el discurso de corte didactista, se muestra más endeble o más contradictorio.

En todo caso, nos aventuramos a apuntar que hay todo un género de estudios de la Educación Física bajo la perspectiva (o alguna perspectiva) inspirada en Foucault que, aunque tal vez en este momento comienza a decaer, ha deja-

Fecha recepción: 07-02-20. Fecha de aceptación: 08-06-20

Miguel Vicente Pedraz

mvicp@unileon.es do cierta impronta que a nuestro juicio merece la pena poner de relieve. Sobre todo, nos parece oportuno revisitar la conceptualización de la «caja de herramientas» de Foucault con el propósito de visibilizar las problematizaciones a las que ha dado lugar en las últimas décadas. Por otra parte, con la perspectiva que ofrece la distancia temporal, nos parece relevante valorar en qué medida -cuánto y para quiénes- la perspectiva foucaultiana ha constituido un recurso de comprensión del campo.

En un trabajo preliminar sobre la obra de Foucault en el campo académico de la educación física en Brasil y Argentina (Pich, \& Rodríguez, 2014) fue analizada, comparativamente, el modo en que fue recibida en sendos países. El mencionado texto se detiene en los alcances y límites de esa apropiación a la vez que muestra cómo los conceptos en ellos invocados (discurso, poder disciplinar y biopolítica) fueron las principales herramientas utilizadas en el campo. Sin embargo, para alimentar el horizonte de posibilidades de las investigaciones pautadas en el referencial teórico foucaultiano, o que utilizan la caja de herramientas foucaultiana en el ámbito de la educación física, entendemos que es relevante retomar y redirigir el análisis haciendo hincapié en la dimensión metateórica de la obra de Foucault; particularmente, en la dimensión del discurso, con el objeto de plantear nuevas perspectivas de comprensión del mismo o, al menos, para abrir nuevas problematizaciones al respecto. Aunque el antecedente de este trabajo esté en la recepción de la obra foucaultiana en el campo académico de la educación física de Brasil, sospechamos que esa esa lectura puede ser extrapolada a los estudios de cuño foucaultiano de la educación física a Iberoamérica en general.

Un primer movimiento que consideramos necesario para ampliar este análisis es considerar la dimensión editorial del 
proceso y su relación con los movimientos del campo académico. A pesar de que Foucault es un autor fallecido hace ya 35 años, su obra todavía es un work in progress. De hecho, un sinnúmero de cursos, escritos, charlas y un libro han sido publicados tras su muerte, en 1984, y con gran probabilidad seguirán publicándose en el futuro. Lo más reciente es el cuarto volumen del proyecto Historia de la sexualidad los secretos de la carne, publicado solamente en francés en 2018 y en castellano en 2019, después de una larga disputa ética, jurídica y económica. Ese carácter escalonado de la aparición de su obra ha consolidado la idea de la existencia de diversos Foucaults, sobre todo, a partir del momento y de los modos en que la obra fue recibida en diferentes dominios del campo académico y en diferentes contextos. Es digno de notar que los cursos dictados en el Collège de France, los cuales hicieron emerger una nueva lectura de Foucault, fueron editados póstumamente. A título de ejemplo, en Brasil comenzaron a publicarse en 1999: primero fue Defender la sociedad y el resto, a intervalos más o menos regulares, hasta 2018 en que se publicó Obrar mal, decir la verdad - la función de la confesión en la justicia. Quedan por salir a la luz los cursos dictados en los Estados Unidos así como el libro Las confesiones de la carne. Particularmente, la recepción de Foucault en Brasil tuvo un carácter ambivalente en virtud del momento en el que se comenzó a leer de manera masiva. A pesar de que Foucault era un asiduo conferencista en el país durante la década de los '70 del siglo XX y un autor bien conocido en el campo de las ciencias humanas, su notoriedad pública solo se elevó considerablemente en el inicio de la década siguiente.

No obstante, incluso a finales de los ochenta, su inserción en el campo de la educación y, sobre todo, en el de la educación física era escasa fruto de la polarización que la reciente redemocratización del país (1985) había generado en el campo; la orientación marxista en los estudios estaba tan arraigada que Foucault era visto con gran desconfianza. Con todo, en el campo académico de la educación física brasileña no llegaría a ser una referencia destacada hasta la primera década del presente siglo y, en cualquier caso, con tal discontinuidad que muchos de los territorios foucaultianos fueron quedando sin explorar. A ello se añade el carácter atomizado, o en cierto modo tópico y convencional, si no mixtificado, de la recepción de su discurso.

Para acometer el trabajo de problematizar el potencial de desarrollo Entwicklungsfähigkeit (Agamben, 2011, p.257) de la apropiación de Foucault en el campo académico de la educación física, tomando como referencia el caso brasile$\tilde{n}^{1}{ }^{1}$ a partir de referencias puntuales ${ }^{2}$-en particular, la categoría de discurso-, nos parece útil partir del concepto de caja de herramientas de Foucault. Nosotros la entendemos más bien como un principio metodológico; es decir, como algo que está conformado por conceptos que no son fijos a lo largo de su trayectoria investigativa, sino que se van manifestando de diferente modo, siempre tensionados a partir del objeto y los terrenos de excavación que están en la pauta de la pesquisa. Pues bien, el concepto de discurso es uno de esas categorías metateóricas vertebradoras de su trabajo cuyos desplazamientos a lo largo de los diferentes momentos de su obra -el ser-saber, el ser-poder y el ser-consigo, según Deleuze (1992)-, se presta al análisis para ofrecer pis- tas sobre qué dominios serían potencialmente fructíferos para el debate en el campo de académico da la educación física; particularmente, para los estudios referenciados en el horizonte de las ciencias humanas. Consideramos oportuno apuntar aquí la diferencia entre el concepto de discurso y el de teoría en la perspectiva foucaultiana. Si el primero apunta a la indisociabilidad entre el saber y el poder, de acuerdo a la lectura que Foucault realiza con base en Nietzsche, la noción de teoría se está referida a un ideal de neutralidad epistemológica y axiológica y de separación radical entre sujeto y objeto que, como sabemos, el pensador galo ponía en cuestión.

\section{La caja de herramientas de Foucault}

Nos parece oportuno traer a colación la afirmación de Foucault en la entrevista que Gilles Deleuze le hiciera en 1972 -publicada bajo el título Los intelectuales y el poder ${ }^{3}$ - a propósito de su modo de proceder investigativo. Gilles Deleuze advirtió sobre el carácter no totalizante de la teoría foucaultiana, así como que debía ser utilizada como una caja de herramientas, en primer lugar, por el intelectual (que así se transformaría de autor en intelectual) y, también por otros. Deleuze se valió de la metáfora de Proust según la cual la teoría (y el arte es parte de ese juego) es una especie de lente que, si no sirve para mirar el mundo y, por lo tanto, para ser y actuar en el mundo de otro modo, debe ser cambiada por otra: «La teoría no se totaliza, se multiplica y multiplica. Es el poder quien por naturaleza opera totalizaciones, y usted [Michel Foucault] dice exactamente: la teoría por naturaleza está contra el poder.» (Deleuze, \& Foucault, 1977, p.81). No obstante, en una entrevista realizada unos años más tarde, en 1977, al ser preguntado sobre la cuestión, apropiándose del concepto elaborado por Deleuze (caja de herramientas), calibra la respuesta y dice:

«La teoría como caja de herramientas quiere decir:

* Que se trata de construir no un sistema sino un instrumento: una lógica propia a las relaciones de poder y a las luchas que se establecen alrededor de ellas.

* Que esta búsqueda no puede hacerse más que gradualmente, a partir de una reflexión (necesariamente histórica en algunas de sus dimensiones) sobre situaciones dadas» (Foucault, 1977, p.173).

El concepto resulta de capital importancia para la discusión que tratamos de desarrollar porque nos permite entender tanto el modo en que Foucault elabora su teoría como los modos en que podemos pensarla y hacer uso de ella.

A este respecto, por una parte, diremos que la teoría foucaultiana tiene una función práctica, no como la aplicación correcta de una idea -de acuerdo con la tradición platónica y cartesiana-, sino en tanto que la actividad del pensar es en sí misma es una práctica que nos permite construir el mundo; actúa como estructura -del pensamientoestructurante-de la realidad-(Vicente, 2010). Además, a diferencia de la tradición antes enunciada, la idea es siempre contingente respecto del actuar de manera que no se puede dilucidar qué hacer a partir de ella. Está y debe estar siempre en potencia de modo que opera como una fuente de la que emergen nuevas posibilidades. La teoría es, entonces, una práctica y una práctica material. 
Por otra parte, Deleuze y Foucault, de forma similar a lo que Theodor Adorno hiciera, nos advierten de la importancia de priorizar el objeto sobre las lentes entendiendo que ni los objetos ni las lentes están «disponibles» a-priori, sino que ocurre una co-producción de ambas instancias a partir de la confrontación del sujeto que investiga con el fenómeno que problematiza. Frente al modo tradicional de pensar, según el cual los objetos y las lentes están siempre disponibles de manera que al sujeto solo le cabe hacer coincidir unas y otros, propone que si las lentes no son apropiadas para mirar el objeto, antes que forzarlo a ser mirado través de las lentes disponibles, el sujeto ha de producir nuevas lentes lo que, de hecho, acaba disolviendo su neutralidad.

Este principio nos proyecta sobre tres consideraciones o retos.

1) En primer lugar, nos lleva a problematizar el uso de las lentes tradicionales. Es corriente que en el campo académico se tiendan a sedimentar modos de hacer con relación a determinados objetos que no son puestos en entredicho. En este caso consideramos que somos invitados a preguntarnos sobre las reglas que han llevado al campo a mirar a determinados objetos con unas lentes y no otras. Por ejemplo, en el campo de la historiografía del deporte sería oportuno preguntarse por qué la historia oficial lo cuenta a partir de una mirada evolutiva y de consagración de los Juegos Olímpicos como la máxima expresión, sin considerar los problemas económicos, culturales y geopolíticos que están envueltos en ese proceso o haciendo apología del bien necesario del progreso. ¿Qué hace que en el estudio comparado de la educación física se ponga el acento en las «pocas» coincidencias antes que en las «muchas» diferencias entre contextos espaciotemporal y culturalmente diversos para legitimar cierta unidad intrínseca o de destino? En el caso particular de Brasil ¿por qué se ha conservado -incluso en las filas más progresistas- el modelo de periodización eurocéntrico cuyos momentos médico-higienista, deportivista, culturalista y de la salud renovada fueron producidos en y para otras latitudes?

2) En segundo lugar, este principio nos induce a cuestionar las tradiciones académicas hegemónicas; es decir, aquellas que tienden a pensar la investigación a partir de un determinado marco teórico para después ajustar (literalmente) a ellos los objetos de la pesquisa. Este es el caso típico tanto de las ciencias biomédicas en la investigación en la educación física que opera a partir de procesos estandarizados bajo el imperio de los protocolos y que resisten, por no decir se niegan, a ser de otra forma. Pero no solo en el campo biomédico; mucho grupos de investigación del campo de las ciencias sociales operan con esta clase de matrices teóricas, como por ejemplo el marxismo (o mejor dicho, un determinado tipo de marxismo ortodoxo) en Brasil y otras tradiciones a partir de una nueva sacralización del método.

3) El tercer desafío al que nos invita el principio enunciado es que no es la teoría lo que debe guiar el proceso investigativo, sino el objeto y su problematización; aquella sirve a este como las herramientas que escogemos de la caja que tenemos y que testamos hasta encontrar la que mejor nos pueda ayudar a resolver el problema abordado. Eso no significa que la herramienta escogida deba ser definitiva, sino solo la más adecuada de entre las encontradas en ese momento entendiendo que puede haber nuevos intentos, nuevas búsquedas y nuevas herramientas en otros momentos. La provisionalidad e historicidad son, entonces, principios clave de este modo de operar.

Un último aspecto de la discusión sobre la caja de herramientas foucaultiana es la dimensión política que el modo de investigación se propone. Al tener la idea de resistencia al poder instituido, como objeto, Foucault parece tener en mente la tradición de la historia social y la historia del pensamiento, contada, ya sea a partir de la derecha (positivismo), ya sea de la izquierda (leyes de la historia), de acuerdo con una lógica determinista y unitaria, que no admite la contingencia o el azar. Es ahí, contra la pretensión totalizante y con aires de necesidad en la forma donde adquiere el poder, donde cobra fuerza la explotación de los saberes locales como estrategia (Foucault se concebía como un estratega). Sin embargo, el carácter local no debe confundirse con una dimensión microsociológica o microhistórica de la realidad; al contrario, lo local tiene un enorme potencial de expansión de acuerdo con, al menos, dos vectores tal y como el pensador galo demostró a partir de la problematización de las prisiones.

1) Por un lado, tiene el poder de multiplicar y legitimar los saberes sobre algo (lo que sin duda trae aparejado el riesgo del relativismo):

«Y cuando los prisioneros se pusieron a hablar, tenían una teoría de la prisión, de la penalidad, de la justicia.

Esta especie de discurso contra el poder, este contradiscurso mantenido por los prisioneros o por aquellos a quienes se llama delincuentes es en realidad lo importante, y no una teoría sobre la delincuencia (...) (Foucault, \& Deleuze, 1977, p.80).

En este caso, el hecho de poner a hablar al Otro implica el reconocimiento de un saber que una teoría totalizante presume como no existente siendo esa presunción, justamente, la que legitima y hace «necesaria» la teoría totalizante como la única válida. Así, los saberes locales y proscritos hacen estallar una miríada de miradas y de mundos que muestran el carácter contingente y en permanente disputa de las relaciones saber-poder.

2) Por otra parte, tiene la capacidad de hacer que el saber local sea el paradigma ${ }^{4}$ de un modo ejemplar de operación del poder:

«Es esto lo que es fascinante en las prisiones, que por una vez el poder no se oculta, no se enmascara, se muestra como tiranía llevada hasta los más ínfimos detalles, poder cínico y al mismo tiempo puro, enteramente «justificado» ya que puede formularse enteramente en el interior de una moral que enmarca su ejercicio: su tiranía salvaje aparece entonces como dominación serena del Bien sobre el Mal, del orden sobre el desorden (Foucault, \& Deleuze, 1977, p.81).

Pero el análisis de la prisión no se circunscribe a la institución en cuanto tal, sino que en ella, según la visión de Foucault, se puede ver el poder operar sin pudores, en su tiranía salvaje, sin máscaras. Lo que significa que la prisión es un caso ejemplar de análisis de los modos de operación del poder que puede servir de paradigma para comprender la naturaleza de las relaciones de poder en la sociedad moderna.

A este respecto, observamos que, para Foucault, el ca- 
rácter político del trabajo intelectual -dicho en términos de Walter Benjamin- consiste en cepillar la historia a contrapelo: ocuparse de ver el poder en su operación, del modus operandi de su circulación, lo que es mucho más potente para desconstruirlo que una crítica moralista en términos de bueno o malo. Esto significa que para poder abrir un horizonte, para el venir-a-ser, se impone en primer lugar no subirse a una tribuna para determinar lo que debería ser y lo que no debería ser, sino tratar de comprender el modo en el cual las relaciones de poder que habitan el mundo moderno tuvieron condiciones de posibilidad. Mostrar su carácter contingente nos coloca también como actores en el juego de unas relaciones de poder que siempre están siendo.

\section{El dominio del ser-saber y el concepto de discurso(s): sobre decires y silencios}

Con estas consideraciones creemos poder abordar el proceso de recepción del concepto de discurso y de análisis discursivo en la educación física brasileña, de acuerdo con la dimensión ser-saber (Deleuze, 1992).

En virtud de que el principal texto referido en los trabajos de la EF brasileña en este dominio es El orden del discurso (Pich, \& Rodríguez, 2014), nos parece oportuno recurrir al mismo para iniciar nuestra argumentación. En la clase inaugural dictada el 2 de diciembre de 1970, la que marca el inicio de su trabajo como profesor del Collège de France, Foucault hace un análisis de las reglas del discurso en la formación discursiva moderna respecto de cuyos principios -el autor, la obra, el comentario y la disciplina- el primero de ellos adquiere un papel central para nuestra argumentación. Foucault presupone que en la moderna producción de las ideas el autor es una función del discurso porque este es producido a partir de reglas de enunciación que no son por él producidas, sino que se enmarcan en un orden discursivo del que él simplemente forma parte. Pero sin duda, el elemento central desde el punto de vista político o de la política de la verdad, como prefería Foucault, es la hipótesis que orienta su trabajo:

«[...] yo supongo que en toda sociedad la producción del discurso está a la vez controlada, seleccionada y redistribuida por un cierto número de procedimientos que tienen por función conjurar los poderes y peligros, dominar el acontecimiento aleatorio y esquivar su pesada y temible materialidad» (Foucault, 1992, p.11-la negrita es nuestra-).

Claramente, aparece señalada la idea de que el control social se ejerce en el plano del discurso y que este, lejos de ser una instancia ideal, es de carácter material; o sea, es en el plano del discurso en donde se encuentra la materialidad de la vida social por lo que el control del discurso equivale al control de la sociedad. El discurso es controlado para evitar que su potencial disruptivo emerja, lo que le confiere un inequívoco valor político. En todo caso, Foucault entiende que es fundamental comprender los modos de control del discurso y, asimismo, entender y dar visibilidad a otras discursividades para hacer surgir fisuras en el discurso oficial.

El trabajo foucaultiano de análisis del discurso está orientado por el proyecto epistemológico-político de carácter crí- tico y genealógico. En su dimensión más crítica se ocupa de las formas del control discursivo y en la genealógica del carácter efectivo de la producción del discurso, aunque evidentemente ambas se muestran indisociables. A ese respecto, se puede decir que las instancias de control están presentes en la producción discursiva y la formación discursiva está conectada con los mecanismos de control como sucede, por ejemplo, en los procesos editoriales y en la crítica literaria:

«La crítica analiza los procesos de enrarecimiento, pero también el reagrupamiento y unificación de los discursos: la genealogía estudia su formación dispersa, discontinua y regular a la vez. A decir verdad, estas dos tareas no son nunca separables; no hay, por una parte, las formas de rechazo, de la exclusión, del reagrupamiento o de la atribución; y después, por otra parte, a un nivel más profundo, el brote espontáneo de los discursos que, inmediatamente antes o después de su manifestación, se encuentran sometidos a la selección y al control. [...] Entre la empresa crítica y la empresa genealógica la diferencia no es tanto de objeto o de dominio como de punto de ataque, de perspectiva y de delimitación» (Foucault, 1992. pp.53-54).

Y a modo de síntesis, concluye:

«En todo caso, una cosa al menos debe señalarse: el análisis del discurso así entendido no revela la universalidad de un sentido, saca a relucir el juego de la rareza impuesta con un poder fundamental de afirmación. Rareza y afirmación, rareza, finalmente, de la afirmación, y no generosidad continua del sentido, ni monarquía del significante» (Foucault, 1992, p.57).

Con todo, el análisis del discurso propuesto por Foucault, además de comportar una sistematicidad que es preciso cuidar, se inscribe en el marco de la disputa por la política contemporánea de la verdad. Lo que se está problematizando en este emprendimiento crítico y genealógico son las claves interpretativas para la comprensión de los modos de control, producción y circulación del discurso; en cierto modo, se está dilucidando cuáles son las reglas y los procedimientos para que aquello que es aceptado como verdadero produzca efectos de verdad a partir de su carácter plural y disperso, no unitario. En ese movimiento se producen tanto dichos como silenciamientos y es ese juego que se trata de comprender.

En el contexto del dominio del ser-saber Foucault asume una perspectiva estructuralista, con reticencias y cierta ironía, cuando en los momentos finales de El orden del discurso dice: «Y ahora, que los que tienen lagunas de vocabulario digan -quizás porque les suena bien- que se trata de estructuralismo» (Foucault, 1992, p.44). De esa forma, a pesar de su posición ambivalente, la concepción del discurso presente puede ser caracterizada como un sistema en el cual el sujeto ocupa una posición o una función que es definida a-priori por el propio sistema; el sujeto pasa a ser un efecto del discurso que domina el tiempo en el que él vive. Por ese motivo, el pensador francés concibe al sujeto moderno en su clásica obra La arqueología del saber como un ser que habla, que vive y que trabaja, de acuerdo con los tres dominios de la ciencia moderna: la lingüística, la biología y la economía. Lo que está en juego es el modo de producción del discurso moderno cuya episteme tiene como eje vertebrador 
la ciencia a la cual mira como la forma privilegiada de expresión del régimen de verdad. $\mathrm{Al}$ analizar los juegos lingüísticos a partir de los cuales se produce no la verdad, sino la veridicción, lo que tiene efecto de verdad, podemos decir que el sujeto es jugado por esas reglas y es el resultado de tales juegos, no participando de la producción de las reglas. A pesar de que Foucault reconoce, como hemos señalado, que lo importante es comprender cómo operan las reglas del juego, es imprescindible abrir posibilidades para otros juegos posibles y para rememorar los juegos olvidados y silenciados, los discursos proscritos y los contradiscursos ${ }^{5}$.

En este punto, consideramos relevante enunciar algunas interpelaciones, aunque provisorias, sobre el campo de la Educación Física brasileña. En primer lugar, nos preguntamos en qué medida el concepto de caja de herramientas ha sido interpretado de manera apropiada y cómo el procedimiento metodológico apuntado por Foucault ha sido puesto en práctica en sus dimensiones crítica y genealógica; si se prefiere, ¿hasta qué punto se ha hecho una interpretación sui generis del método? Nos preguntamos también ¿en qué medida el carácter conformador de la vida social que atribuye al lenguaje ha sido adecuadamente problematizado y puesto en juego en el contexto específico del lenguaje corporal?; en todo caso, ¿cómo han sido producidas y cómo operan las reglas del discurso del lenguaje corporal en la modernidad? Por otro lado, también nos preguntamos ¿en qué medida las reglas discursivas que operan en el juego de lo verdadero y lo falso de la educación física han sido cuestionadas a partir del referencial foucaultiano, involucrando lo dicho -que pasa a ser verdadero-y lo no dicho-lo silenciado o proscrito, los contradiscursos-? ¿Se ha preguntado la educación física sobre los elementos que conforman el orden de discurso, a saber: sobre los objetos elevados al rango de tabú, los rituales de circunstancia que determinan los modos legítimos de decir y el derecho exclusivo o privilegiado de los sujetos autorizados a decir?

Valga subrayar en este momento el cuestionamiento de los nuevos dominios del saber y cómo estos pugnan por decir qué es el cuerpo cuya influencia en el campo profesional de la educación física es evidente. El cuerpo, de acuerdo con Le Breton (2002), está sujeto a los avatares del desarrollo tecnológico y adquiere con el tiempo prótesis mecánicas, injertos, tatuajes, es objeto de la ingeniería genética, es recreado o duplicado virtualmente para las redes sociales y en los juegos electrónicos, hasta el punto de que la tecnología y lo virtual contribuyen hoy la materialidad del cuerpo; una materialidad que hace de él un borrador en el que se escribe constantemente, llevando a la producción de otras metáforas corporales como ha señalado Vilanou (2000). En ese sentido, parece que el discurso gana un carácter efímero que es preciso comprender y tal vez sea un modo válido de cuestionamiento de la propia categoría.

En este plano de debate vale la pena destacar el movimiento que ha experimentado la producción científico-académica del sub-campo de las ciencias humanas en el ámbito de la educación física brasileña en los últimos años. A partir de la disputa entre las ciencias humanas (lo que en Brasil se ha denominado de la sub-área área sociocultural y pedagógica) y las ciencias biomédicas (que lleva el nombre de biodinámica) se observa, de acuerdo con Rigo, et al. (2011), una creciente concentración de la producción y de los investigadores de la segunda área en los programas de post-grado en educación física en Brasil. El modo de jugar con las reglas de la producción científica ha alejado a los investigadores del ámbito de las ciencias humanas del círculo específico de la producción científica brasileña, lo que ha llevado a una recuperación de la hegemonía del saber biomédico en la producción de la identidad académica de la Educación Física (saberes considerados legítimos). Esa constatación fue complementada en un trabajo realizado por Pich, et al. (2015) en la que se identificó que de los dieciséis investigadores con publicación constante en el Grupo de Trabajo de Epistemología (GTT-4) del Colegio Brasileño de Ciencias del Deporte (CBCE) trece no desarrollaban su trabajo en programas de post-grado en Educación Física. Esta circunstancia podrá llevar en un futuro próximo a que los profesorados de educación física, cuyos profesores tengan una identidad académica y pedagógica con la concepción (neo)biologicista de la educación física, tengan una impronta de tal concepción en el proceso formativo, dado que los docentes de grado son egresados de los programas de post-grado (Pich, 2014). Apuntamos, además, que ese proceso no se realiza sin resistencia en foros como el antes mencionado Colegio Brasileño de Ciencias del Deporte (CBCE), pero que, desde el en la implementación de políticas públicas de producción de conocimiento en la Educación Física, se observa un claro en avance de las áreas biomédicas sobre las ciencias humanas. En ese breve cuadro de situación podemos ver como la lucha por definir las reglas del discurso opera fuertemente en el campo y está teniendo en el momento presente un impacto considerable en los modos de producción de un campo de saber/hacer a partir de la institución de aquello que es legítimo decir y aquello que debe ser silenciado. Algo parecido ha sucedido en España, según lo pusieron de relieve Vicente, \& Brozas (2014) a propósito de la evolución de los planes de estudio en el ámbito de la educación superior de Educación Física.

En otro plano, ahora más particular, destacamos un hecho que podría ser pensado en la clave teórica que analizamos. Un joven profesor de Educación Física, el Prof. William Meister, con el cual hemos tenido una fructífera relación académica y que trabajaba en una escuela municipal de enseñanza, vio interrumpido su contrato a raíz de una denuncia hecha por los padres de un alumno porque había utilizado una poesía sobre la tortura durante la dictadura militar para explicar los mecanismos de control sobre los cuerpos en el contexto en el que la educación física había asumido un carácter deportivista ${ }^{6}$. Lo que Foucault puede aportar al análisis de este caso es una forma de mirar sobre cómo operan los modos de control; cómo a través de la formación y la afirmación del discurso es posible desvelar tabúes, determinar cuáles son los comentarios pertinentes y quiénes los sujetos autorizados a enunciarlos.

Haciendo referencia al campo académico de la educación física brasileña, la primera elaboración del concepto de discurso en Foucault ha seguido recibiendo atención, afinando el uso de esta herramienta para diversos y nuevos objetos. Destacamos algunos textos que ilustran este movimiento que permanece y gana más densidad en la operación con la categoría en cuestión. El primero que traemos a cola- 
ción es el texto de Rigo y Santolin (2015) que lleva el título de «O nascimento do discurso patologizante da obesidade». Vale recordar que Rigo fue uno de los autores discutidos en el trabajo anterior que dio base a este texto. En ese trabajo se observa un cuidadoso uso del análisis del discurso foucaultiano para pensar la obesidad y los enunciados que caracterizan su medicalización y la consecuente emergencia en el dominio biomédico así como, también, las implicaciones políticas, sociales y culturales de ese fenómeno. Otro trabajo que merece ser destacado aquí es el de Alex Branco Fraga y Giliane Dessbesell, titulado «Exercícios físicos na Base Nacional Comum Curricular: um estranho no nicho da cultura corporal de movimento». En este caso observamos una interesante elaboración de la noción de discurso foucaultiana para discutir la economía del campo discursivo de la Educación Física escolar brasileña y las vicisitudes de los actores de la corriente culturalista para tratar con un objeto como el de los ejercicios físicos, que se encuentra umbilicalmente ligado al conjunto de signos que identifican el discurso de la corriente biologicista de la salud y la aptitud física. En los casos citados a modo de ejemplo se puede observar tanto la presencia del concepto de discurso en su primera elaboración y el análisis de discurso que emerge de ese concepto, están operando con vigor en el campo de la educación física y demostrando su potencial como herramienta analítica para nuevos problemas del mismo ${ }^{7}$.

\section{La pragmática en el discurso de Foucault}

Como ya señalamos anteriormente, el concepto de discurso más citado en la producción del campo académico de la educación física brasileña es el que se hace presente en el texto El orden del discurso que el pensador galo elaborara en la década de los sesenta, en particular, en Las palabras y las cosas y en La arqueología del saber. Sin embargo, dicha noción, no permanece inalterada a lo largo de su obra. En la medida en que fue explorando nuevos objetos y ámbitos iría revisando y adecuando las herramientas con las que operaba. Si algún principio se mantuvo más o menos fijo es que el lenguaje no es de carácter representacional, sino que es en el lenguaje en donde el mundo se da; noción tributaria del giro lingüístico en el que de alguna forma Foucault se instala. A este respecto, la manera de concebir el concepto de discurso tiene diversos desplazamientos que, según nuestra matriz analítica, son correlativos al modo en que Foucault avanza en los diferentes dominios de su obra: el ser-saber, el serpoder y el ser-consigo (Deleuze, 1992).

En el segundo de estos dominios, el del ser-poder -más conocido como la genealogía del poder- acomete nuevamente la cuestión del discurso. El problema está planteado desde el año 1973 durante las conferencias que Foucault dictó en la Pontificia Universidad Católica de Río de Janeiro (PUC/RJ) -publicadas por primera vez en 1978 en el libro La verdad y las formas jurídicas (Foucault, 1996) y que serían ampliadas a lo largo de esa década e inclusive en la década siguiente-. En este caso el autor se preocupa no tanto por la producción del discurso en el dominio de las ciencias, como por la manera en que los sujetos producen cosas con palabras a partir de contextos determinados de acción lingüística en cuyos discursos son interpelados (Foucault, 1996).
Se percibe aquí la presencia de la idea de performatividad del lenguaje que establece que las palabras no representan cosas sino que las producen -dadas determinadas condiciones de enunciación, por lo general de carácter ritual-, influido por la pragmática del discurso de John Langshaw Austin en su clásica obra How to do things whit words (¿Cómo hacer cosas con palabras?) cuya publicación data de $1962^{8}$. Así, por ejemplo, en La verdad y las formas jurídicas Foucault plantea que cuando un juez abre una sesión de un juicio, al decir está abierta la sesión no está reflejando ninguna realidad exterior a las palabras, sino instituyendo la sesión a partir de un acto lingüístico (speech act). Particularmente, interpela a Foucault la importancia que con respecto al sistema penal tiene el acto del reo al declararse culpable (o inocente): ¿por qué es tan importante el acto en el cual el acusado se reconoce como culpable? Según Foucault (1996) porque es justamente en ese acto en el cual el sujeto deviene culpable y encuentra y realiza en sí mismo su realidad de sujeto criminal. Además, el sistema penal (a partir de su herencia cristiana) instituye en el acto de reconocimiento de la culpa el momento en el que es posible iniciar la restitución del sujeto a la normalidad de la vida social, su proceso de recuperación o resocialización. Pero este ritual, repetimos, debe ser realizado en un contexto institucional en el cual los actores están investidos de la legitimidad que el status social les confiere, no importando la condición ética del sujeto sino, como diríamos apelando a Weber, el carisma de cargo. Ese estudio se enmarca en el contexto de la investigación de las tecnologías del yo (Foucault, 2008) -desde mediados de los setenta- y que sería sistematizado en la conferencia Las tecnologías del yo, dictada en 1981 y publicada en 1988 como parte del libro homónimo.

Entendemos que este modo de análisis tiene tanta potencia para problematizar fenómenos del campo de la Educación Física como para otros campos donde ya se ha revelado muy fecunda. Si nos valemos del modelo presentado por Foucault para el campo jurídico, nos podemos preguntar ¿de qué forma el profesor de educación física, como representante de un dominio de saber, normalmente referenciado en el ámbito de las ciencias biomédicas, opera en el manejo de sus actividades en un patio de escuela, en un centro de entrenamiento deportivo o en un gimnasio de musculación, por citar algunos ámbitos de intervención profesional? Al orientar prácticas corporales -o sería más adecuado en este momento decir de actividades físicas- ¿los profesores de educación física instituyen y sustancializan la condición adscrita sus alumnos, al mismo tiempo que también los llevan a reconocerse y a instituirlos como, por ejemplo sujetos sedentarios, obesos, descoordinados, con talento, etc.? Decir eres obeso o eres sedentario o tienes talento, a pesar de ser presentado como una mera constatación de la verdad medida por las diferentes baterías de evaluación antropométricas y de la condición física, adquiere en este registro teórico, no un carácter representacional, sino enteramente creador; opera como un modo de producción del sujeto a partir de su vinculación con la condición enunciada. Dicho de otro modo, a partir del rol social que desempeña y del estatus social que le es atribuido el profesor como representante de un saber legítimo, a través de ciertos rituales adheridos a dicho saber, lleva al otro, a quien dirige en sus prácticas profesionales, a 
identificarse según parámetros de normalidad conformados por las ciencias biomédicas (Vicente, 2001), asumiendo así una bioidentidad (Ortega, 2003). Además, en ese contexto el proceso de orientación de la actividad física o del entrenamiento corporal, que es iniciado a partir de la enunciación del sujeto de su condición, sigue el formato del camino de expiación de la culpa que es asumida conjuntamente con la identidad en el momento de dicha enunciación.

Dialogando con el campo brasileño de la Educación Física, el segundo momento de elaboración del concepto de discurso, situado en el contexto del dominio del ser-poder (o de la genealogía del poder) tiene interesantes trabajos a ser apuntados a modo de ejemplo. Destacamos de entre ellos el de Carmen Lúcia Soares y Jaques Gleice que lleva por título «Como se fabricam os anjos? Uma arqueologia do corpo nos manuais escolares de moral e de higiene na França, 18801974». En ese texto observamos un interesante análisis de manuales escolares para definir un cuerpo inscrito en un régimen de moralidad heredero de la tradición judeo-cristiana, del cual la escuela «laica» francesa es su locus de producción y reproducción. La escuela es concebida como el nuevo templo de producción de cuerpos moralizados e moralizantes a partir de los rituales escolares orientados por la moral cristiana secularizada (Soares, \& Gleice, 2012). También vale la pena señalar el estudio realizado por Fabio Zoboli, et al. (2020), intitulado «Dilemas heteronormativos de feminilidade em exemplares da Revista Playboy onde posaram atletas». Es un brillante trabajo que muestra, en perspectiva post-estructural, la política de verdad con relación al imperio del régimen moral heteronormativo en las ediciones de la versión brasileña de la revista Playboy en las cuales son retratadas mujeres deportistas. En ese trabajo los autores muestran el modo en que los enunciados relacionados con el régimen moral heteronormativo se imponen para producir la materialidad textual de la imagen, aliados a un ritual imagético de presentación y realización del ideal femenino. En ambos trabajos vemos la idea de la importancia de un contexto ritual (físico, como en el caso de la escuela, o imagético, como en el caso de la Revista Playboy) en el cual se inscriben las palabras que definen e instituyen el orden de lo real, produciéndolo de modo eficaz ${ }^{9}$. Estos ejemplos, unido a lo que apuntamos anteriormente, nos muestran que esa matriz que puede ser un rico horizonte para orientar investigaciones en el campo de la educación física en Brasil, pero también en otros contextos.

\section{El tercer desplazamiento: la dramática del discurso}

La noción de discurso experimentaría un nuevo desplazamiento en el momento en que el pensador galo empieza a ocuparse de la genealogía de la moral, o del ser-consigo, ya en la década de los ochenta, cuando ya tiene como par conceptual vertebrador al cuidado de sí y la parrhesía ${ }^{10}$.

Siguiendo la lectura deleuziana, al operar el pliegue de sí sobre sí mismo, la subjetividad emerge como obra de arte; la relación del sujeto con la palabra cambia sustancialmente porque tiene lugar un proceso artesanal y cuidadoso de producción e incorporación (en el sentido de volverse cuerpo, de una palabra hecha cuerpo). En cualquier caso, el cuerpo y la inmanencia que este implica forman un par conceptual fundamental para comprender este modo de producción de la palabra: el sujeto pasa a vincularse éticamente con la palabra y a moldear su vida a partir de ella, a producir una estilística de la existencia, lo que implica que el acto de enunciación es un acto de producción de la subjetividad y no de sumisión del sujeto a un régimen de moralidad definido $a$ priori y de carácter heterónomo, como en las situaciones del ser-saber y del ser-poder tanto en la función autor como en el enunciado performativo, respectivamente. Si hasta el momento anterior la relación con la palabra marcaba principalmente una relación de sujeción, con la dramática del discurso, en el ser-consigo, irrumpe algo totalmente nuevo o, al menos, embarazoso: cuidar de sí tiene algo de perturbador (Foucault, 2006). Eso totalmente nuevo solo deviene a partir de una relación de habla franca con el otro, de la parrhesía donde la relación parresiástica es, por definición, una relación de riesgo; riesgo de quien habla porque compromete la relación con el Otro al decirle audazmente la verdad, y riesgo del escuchante porque la verdad de sí es siempre incómoda para aquel que está acostumbrado a una imagen narcisista; porque cumple el rol de decir algo sobre una región oscura de sí, a la que en soledad no se tendría acceso (Foucault, 2006).

Uno de los pasajes que vale la pena recordar en ese sentido es la interpretación que hace Foucault de la relación de Sócrates con el bello joven Alcibíades. Sócrates era el único que no le buscaba para obtener favores sexuales, sino que sólo se aproximaba de él con la intención de decirle la verdad de lo que era y lo que precisaba para volverse un líder político de Atenas. Sócrates interpelaba a Alcibíades con el objeto de que entendiera que para poder cuidar de los otros (gobernar la ciudad), debía primero aprender a cuidar de sí (gobierno de sí), poniendo en riesgo la relación entre ambos (Foucault, 2006). Las palabras de Sócrates eran de hecho incómodas para el joven Alcibíades; pero actuando de esa manera Sócrates le estaba demostrando su verdadero amor, a pesar de que al final del diálogo (Primer Alcibíades) el maestro de la mayéutica desconfíe de las posibilidades de realización del proyecto formativo de su discípulo, no tanto por desconfiar de la naturaleza de él, como por la incapacidad del pueblo ateniense (marcada por el vicio). Ese carácter disruptivo de la palabra tiene por objeto hacer nacer otro de sí mismo, donde ese otro carece de modelo previamente fijado, es fruto de una elaboración de la propia vida a partir de la relación amorosa con el Otro, el maestro. En efecto, de eso trataba la mayéutica: de llevar al sujeto a hacer un trabajo de parto, a hacer nacer a otro desde sí.

El planteamiento nos coloca ante un cierto desdoblamiento de la función del discurso que Foucault desarrolló en la clase que impartió el 12 de enero de 1983 como parte del curso El gobierno de sí y de los otros (Foucault, 2009). En ella diferenciaba entre la pragmática y la dramática del discurso a partir de tres condiciones: 1) la previsibilidad del efecto del enunciado; 2) la relación del sujeto con la enunciación y 3) la posición que confiere valor de verdad al enunciado. Veamos esquemáticamente cada uno de ellos.

En primer lugar, el enunciado performativo es altamente codificado y su efecto es claramente previsible, por ejemplo en el bautismo. Cuando un sacerdote administra el sacramento debe pronunciar determinadas palabras yo te bautizo 
en nombre del Padre, del Hijo y del Espíritu Santo y realizar determinados gestos, de lo cual solamente podrá resultar el hecho del ingreso de un sujeto al universo del cristianismo. Por otro lado, el enunciado parresiástico habla para un horizonte abierto, del cual no se saben las consecuencias: «la irrupción del discurso verdadero determina una situación abierta o, mejor, abre la situación y hace posible una serie de efectos que, justamente, son desconocidos. La parrhesía no produce un efecto codificado: abre un riesgo determinado» (Foucault, 2009, p.78).

En segundo lugar, la relación del sujeto con el enunciado se define a partir una diferencia ética en esa relación. En el enunciado performativo es condición de posibilidad para su eficacia que el enunciador tenga un estatus socialmente legitimado, pero no lo es que se vincule éticamente con el enunciado; no hay ninguna necesidad de que al decir te pido perdón el acto lingüístico implique al sujeto en aquello que es dicho para que el acto del perdón se realice. En el caso del enunciado parresiástico la vinculación ética del sujeto con el enunciado es condición de posibilidad para devenir verdadera. Es lo que se denomina pacto parresiástico, que no es un pacto con otro, sino consigo mismo.

«La parrhesía, pues, abarca el enunciado de la verdad y, además, por encima de ese enunciado, un elemento implícito que podríamos llamar pacto parresiástico del sujeto consigo mismo, en virtud del cual éste se liga al contenido del enunciado y al propio acto de enunciarlo: soy quien habrá dicho esto» (Foucault, 2009, p.81).

A este respecto, la palabra que imparte es una dimensión indisociable de su vida, una marca de su virtud y por ese motivo se compromete y se implica éticamente con ella, asumiendo el riesgo que ello presupone. El discurso parresiástico trata del saber de otro saber, del saber que se inscribe en la vida y no de un saber con el que se guarda una relación de exterioridad, una relación instrumental en terminología frankfurtiana.

Por lo que se refiere a la tercera condición, en el caso del enunciado performativo es necesario que el sujeto tenga un estatus socialmente reconocido para que al pronunciar el enunciado, realice la cosa dicha. En situaciones corrientes como en el caso de perdonar, es necesario haber sido ofendido para poder realizar el acto lingüístico del perdón. En otro registro, el enunciado parresiástico opera sin que haya condición de estatus social para que este tenga validez. En palabras de Foucault: «La característica del enunciado parresiástico es justamente que, al margen del estatus y de todo lo que pueda codificar y determinar la situación, el parresiasta es quien hace valer su propia libertad de individuo que habla» (Foucault, 2009, p.81). De esa manera, no hay una condición necesaria que vuelva posible la enunciación, sino que esta emerge a partir de la relación ética del sujeto con su vida y la de los Otros. Además, la autoridad del parresiasta se origina en su modo ejemplar de vida, en la armonía entre su palabra y su modo de vivir.

Con todo, se puede colegir que aquello que Foucault denomina dramática del discurso está umbilicalmente ligado a la parrhesía, al enunciado parresiástico, cuyo objeto es la transformación del ser del sujeto. Vale la pena recuperar en este momento el concepto de parrhesía de Foucault por su carácter metodológico:
«La parrhesía (...) es pues cierta manera de hablar. Más precisamente, es una manera de decir la verdad. En tercer lugar, es una manera de decir la verdad de tal modo que, por el hecho mismo de decirla, abrimos, nos exponemos a un riesgo. Cuarto, la parrhesía es una manera de abrir ese riesgo ligado al decir veraz al constituirnos en cierta forma como interlocutores de nosotros mismos cuando hablamos, al ligarnos al enunciado y la enunciación de la verdad. Para terminar, la parrhesía es una manera de ligarnos a nosotros mismos en la forma de un acto valeroso. Es el libre coraje por el cual uno se liga a sí mismo en el acto de decir la verdad. E incluso es la ética del decir veraz, en su acto arriesgado y libre» (Foucault, 2009, p.82).

Es definitiva, con la dramática del discurso, Foucault está diciendo que los hechos discursivos muestran de qué manera el acontecimiento mismo de la enunciación puede afectar el ser del que enuncia (Foucault, 2009, p.84).

Pensando en el campo de la educación física, esta conceptualización nos insta a analizar lugares todavía inexplorados y confesamos que en este caso tenemos más dudas que certezas. Entendemos que es oportuno interrogarnos sobre el registro discursivo que impera en el campo: ¿todavía estamos presos en una pragmática del discurso, por tanto, en procesos de formación pautados por una relación formal en la cual aquello que es dicho no toca al ser del sujeto y se enmarca en contextos institucionales altamente codificados? o, por el contrario ¿se observan instancias en las cuales aquello que es dicho emerge de la condición ética del sujeto?; ¿estamos priorizando aún la expertise técnicoprofesional o se han dado ya pasos para que la formación tenga como objeto la constitución ética de los profesores aprendices? Esto de ningún modo significa negar cualquier valor de verdad a saberes de origen científico, sino que alerta de la necesidad de pensar nuestra relación con esos saberes. Por otro lado, también nos cuestionamos si sería posible pensar en la naturaleza de los saberes de lo corporal a partir de este registro teórico: ¿si el cuerpo ha sido y continúa siendo dicho (solo) por la ciencia y la técnica? ¿Qué sería la ejemplaridad y la virtud en el campo de la educación corporal? ¿Qué significa arriesgarse o ponerse en riesgo en la relación formativa en el campo de la educación corporal? O más, ¿sería adecuado seguir hablando de educación corporal en tanto que posibilidad para la enunciación? En fin, cuando nos faltan las palabras o se enrarecen es que estamos explorando los límites de lo que somos para tornar como posible otra realidad, otros devenires.

Indagando la producción en el campo académico brasileño de la educación física, con relación a este dominio de la obra foucaultiana, el ser-consigo, nos confrontamos con una escasa producción, pero que da cuenta de que el mismo está teniendo resonancia en la producción del campo. Ilustramos este movimiento a partir de dos textos. El primero es intitulado «O yoga como prática de áskesis», de autoría de Rabello, et al. (2018). En él podemos ver un interesante ejercicio en el que serelaciona la práctica del yoga con la noción de áskesis, categoría central por el pensador galo al analizar los escuelas del helenismo griego y romano. En ese análisis opera diálogo entre la comprensión de la relación entre el maestro y el discípulo y el problema ético implicado en los dos registros 
analizados. Otra referencia que merece mención es el texto «A 'escrita de si' na formação em Educação Física» de Carvalho, et al. (2011). En este caso el acento está puesto en la importancia de la escritura sobre la práctica pedagógica del profesor de educación física en proceso (permanente) de formación, que sobrepasa los límites de aquello a lo cual el conocimiento formal de la ciencia permite acceso y, en ese sentido, tiene potencia para lidiar con la vida y su polifonía en su permanente devenir. Señalamos, además, la centralidad que tiene en los dos trabajos citados el problema de la constitución ética del sujeto, a partir de la práctica permanente de símismo.

\section{Consideraciones finales}

Acercándonos al final de nuestra exposición entendemos importante resaltar la idea de que no existe El Discurso (con mayúsculas) en la obra de Foucault, unívoco, sino discursos; que el discurso es polifónico y que, como categoría del pensamiento o herramienta teórica, es revisada a partir del objeto y de los terrenos de excavación en los cuales el autor se aventura. La idea de caja de herramientas es, así, un principio metodológico del trabajo del pensador galo, puesto que los conceptos no operan de manera a-priorística con relación a los objetos, sino a partir de ellos. De ese modo, parece corroborarse la hipótesis que hemos sustentado; a saber, que en los diferentes dominios foucaultianos -sersaber, ser-poder y ser-consigo- no hay una sino diferentes elaboraciones del concepto de discurso.

Al dialogar con el campo académico de la educación física brasileña sobre el modo de apropiación de esta categoría, vemos que la obra foucaultiana continua presente y posibilitando una amplia gama de posibilidades investigativas revelando así, la multivocalidad y multiplicidad del campo. A pesar de ello, el dominio del ser-consigo, continúa siendo prácticamente inexplorado. De ese modo entendemos que el concepto de discurso, lejos de haberse agotado, exhibe un gran potencial de desarrollo (Agamben, 2010) para pensar el campo de la educación física. Buena parte de cuanto contiene la caja de herramientas de Foucault todavía está con poco uso (o ninguno) y prometen un rico horizonte para pensar lo que venimos siendo, así como también devenires posibles.

\section{Referencias}

Agamben, G. (2010). Signatura rerum; sobre el método. Madrid: Anagrama.

Carvalho, et al. (2011). A «escrita de si» na formação em Educação Física. Movimento, Porto Alegre, 17(02),239258.

Austin, J. L. (1962). How to do things with words. Londres: Oxford University Press.

Cassani, J. M. (2020). Práticas Científicas em Circulação no GTT Memórias da Educação Física e Esporte do Colégio Brasileiro de Ciências do Esporte (2005-2017). Retos. Nuevas tendencias en Educación Física, Deporte y Recreación, 38,472-481.

Santolin, C. B., \& Rigo, L. C. (2015). Movimento, Porto Alegre, 21(1),81-94.

Deleuze, G. (1992). Conversações. São Paulo, Editora 34.
Dessbesell, G., \& Fraga, A. B. (2020). Exercícios físicos na Base Nacional Comum Curricular: um estranho no nicho da cultura corporal de movimento. Movimento, Porto Alegre, 26, e26007.

Foucault, M. (1977). Microfísica del poder. Madrid: La Piqueta.

Foucault, M. (1992). El orden del discurso. Buenos Aires: Tusquets Editores,

Foucault, M. (1996). La verdad y las formas jurídicas. Barcelona: Gedisa.

Foucault, M. (2003). Historia de la Sexualidad, 2. El uso de los placeres. Buenos Aires: Siglo XXI.

Foucault, M. (2006). La hermenéutica del sujeto. Buenos Aires: Fondo de Cultura Económica.

Foucault, M. (2008). Las tecnologías del yo. Buenos Aires: Paidós.

Foucault, M. (2009). El gobierno de sí y de los otros: curso en el Collège de France. Buenos Aires: Fondo de Cultura Económica.

Foucault, M., \& Deleuze, G. (1977). Los intelectuales y el poder. Madrid: La Piqueta.

Góis Jr., E., et al. (2014). O discurso médico e a Educação Física nas escolas (Brasil, século XIX). Bras. Educ. Fís. Esporte, São Paulo, 28(2), 273-82.

Le Breton, D. (2002). La sociología del cuerpo. Buenos Aires: Nueva Visión.

Ortega, F. (2003). Práticas de ascese corporal e constituição de bioidentidades. Cadernos de Saúde Coletiva, 11(1),59-77.

Pich, S. (2014). La formación de formadores y de profesores de Educación Física en Brasil: la tensión entre la producción de conocimiento y la identidad epistemológica. Camblor, E., et al. (dir.) Prácticas de la educación física (pp. 15-26). La Plata: Editorial de la Universidad Nacional de La Plata (UNLP).

Pich, S., \& Rodríguez, N. (2014). Los cuerpos de Foucault: una genealogía de los estudios foucaultianos en el campo de académico de la Educación Física en Brasil y en la Argentina. Revista Brasileira de Educação Física e Esporte, 28(3),453-67.

Rabello, G. E., et al. (2018). O yoga como prática de áskesis. Motrivivência, Florianópolis, 30(55),208-226.

Pich, S., et al. (2015). Análise da produção dos artigos publicados nos anais do GTT de epistemologia nos CONBRACE/CONICES 2009, 2011 e 2013. Rechia, S., et al. (coord.) Dilemas e desafios da Pós-Graduação em educação física (pp. 505-516). Ijuí: Unijuí.

Rigo, L. C., et. al. (2011). Unidade na diversidade: desafios para a Educação Física no século XXI. Revista Brasileira de Atividade Física \& Saúde, 16(4),339-345.

Soares, C. L., \& Gleice, J. (2012). Como se fabricam os anjos? Uma arqueologia do corpo nos manuais escolares de moral e de higiene na França, 1880-1974. Rev. Bras. Ciênc. Esporte, Florianópolis, 34(4),805-824.

Vicente, M. (2001). La mirada del otro: Escuela, cuerpo y poder. Revista de Educación Física, 84,5-14.

Vicente, M. (2010). Educación física e ideología: creencias pedagógicas y dominación cultural en las enseñanzas escolares del cuerpo. Retos. Nuevas tendencias en Educación Física, Deportes y Recreación, 17,76-85. 
Vicente, M., \& Brozas, M. P. (2014). Evolución de los planes de estudio de Educación Física y Ciencias de la Actividad Física en la Universidad de León: estudio de un (fra)caso. Retos. Nuevas tendencias en Educación Física, Deporte y Recreación, 26,101-107.

Vilanou, C. (2000). La configuración post-moderna del cuerpo humano. Movimento, 13(2),81-98.

Zandoná Jr. A., \& Carneiro, F. H. S. (2018). Reflexões acerca do currículo e metodologia da Educação Física no Instituto Federal de Goiás: a experiência com materiais didáticos. Retos. Nuevas tendencias en Educación Física, Deporte y Recreación, 34,337-342.

Zoboli F. et. al. (2020). Dilemas heteronormativos de feminilidade em exemplares da Revista Playboy onde posaram atletas. Motrivivência, Florianópolis, 32(61),0122.

\section{(Footnotes)}

${ }^{1}$ Entendemos que el campo es múltiplo e diverso en sus posiciones y usos de las herramientas conceptuales. A título de ejemplo destacamos el trabajo de Cassani, et al. (2020) sobre la diversidad de la producción, de grupos y problemáticas, en el dominio de la historiografía de educación física brasileña.

${ }^{2}$ Para este fin hemos consultado las principales revistas del campo académico de la educación física brasileña (usando como criterio la evaluación del Qualis Capes), a saber: Revista Brasilira de Ciências do Esporte (RBCE), Revista Movimento, Revista Brasileira de Educação Física e Esporte (RBEFE), Revista Motrivivência, Revista de Educação Física/UEM e a Revista Motriz y extraído de ellas las producciones más relevantes para el diálogo que proponemos, siendo que fueron seleccionados textos de las 4 primeras, teniendo como criterios para la inclusión en este trabajo: 1) Consistencia de los trabajos y originalidad y multiplicidad de los objetos; 2) Representatividad de los autores en el campo de la Educación Física, en cuanto a importancia y a diversidad de posiciones en el campo en términos epistemológicos y de áreas de estudio. La consulta fue realizada a partir de los descriptores: discurso; Foucault; y discurso and Foucault.

${ }^{3}$ En castellano el texto fue publicado en 1977 en el núme- ro 6 de la revista «El viejo topo» y como parte del libro «Microfísica del poder» que editó La Piqueta.

${ }^{4}$ Aquí operamos con el concepto de paradigma elaborado por Giorgio Agamben, para quien el paradigma es un caso que retirado de la serie, puede arrojar luz sobre toda la serie (Agamben, 2010).

${ }^{5}$ También vale la penar apuntar que lo discursivo y lo no discursivo van a articularse en el concepto de dispositivo que Foucault sistematiza en la entrevista denominada Sobre la historia de la sexualidad, que fue publicada en Brasil como parte de la nueva versión de la Microfísica del poder en 1979.

${ }^{6}$ Véase: https://www.cartacapital.com.br/educacao/scprofessor-e-demitido-apos-trabalhar-texto-sobre-tortura-naditadura-militar-2/page/37/

${ }^{7}$ Es relevante destacar los esfuerzos que vienen siendo realizados en el campo de la educación física escolar, que muestran que también los contra-discursos son posibles y que de ello resulta una nueva comprensión de la educación física, no más en llave de lectura biologicista. Por ejemplo, señalamos la sistematización hecha por Zandoná Jr., \& Carneiro (2018) sobre el currículo de educación física en el Instituto Federal de Goiás (IFG).

${ }^{8}$ Conferencia dictada por Austin em 1955 (Austin, 1962).

${ }^{9}$ En este dominio también merece ser apuntado el excelente trabajo de Gois, et al. (2014) sobre el discurso médico y la educación física en Brasil en el siglo XIX (y el comienzo del siglo XX). A pesar de que no está referenciado en la matriz foucaultiana, tiene gran potencial de diálogo con los estudios foucaultianos arqueogenealógicos sobre el poder biomédico sobre la vida, en particular en la transición del siglo XIX al siglo XX.

${ }^{10}$ Aparece en los cursos dictados en el Collège de France en El gobierno de los vivos 1979-1980; en Subjetividad y verdad 1980-1981; en La hermenéutica del sujeto 19811982 en El gobierno de sí y de los otros 1982-1983 y en El gobierno de sí y de los otros II: el coraje de la verdad 19831984. Asimismo, aparece en los cursos dictados en los Estados Unidos y en Bélgica, en los volúmenes 2 y 3 de la Historia de la sexualidad y en diversos artículos, conferencias y entrevistas que tratan del asunto.
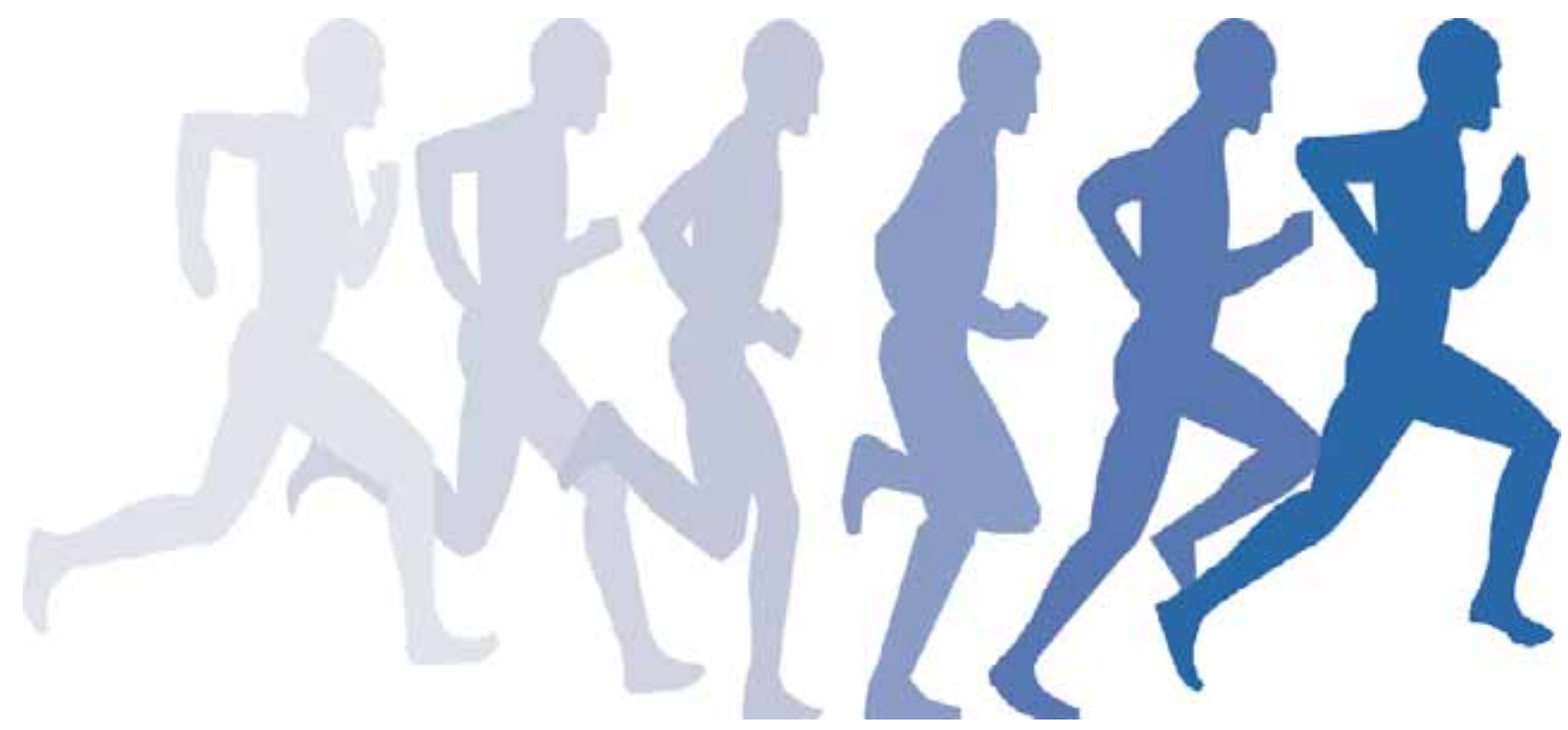